\title{
Surface Chemical Trapping of Optical Cycling
}

\section{Centers}

Han Guo ${ }^{l}$, Claire E Dickerson ${ }^{l}$, Ashley J. Shin ${ }^{l}$, Changling Zhao ${ }^{2}$, Timothy L. Atallah ${ }^{l}$, Justin R. Caram $^{l *}$, Wesley C. Campbell ${ }^{*}$, Anastassia N. Alexandrova ${ }^{1 *}$

${ }^{1}$ Department of Chemistry and Biochemistry, ${ }^{2}$ Department of Physics and Astronomy, University of California, Los Angeles, Los Angeles, CA 90095, USA

KEYWORDS optical cycling, molecular qubits, surface patterning, theory

ABSTRACT Quantum information processors based on trapped atoms utilize laser-induced optical cycling transitions for state preparation and measurement. These transitions consist of an electronic excitation from the ground to an excited state and a decay back to the initial ground state, associated with a photon emission. While this technique has been used primarily with atoms, it has also recently been shown to work for some divalent metal hydroxides (e.g. $\mathrm{SrOH}$ ) and alkoxides (e.g. $\mathrm{SrOCH}_{3}$ ). This extension to molecules is possible because these molecules feature nearly isolated, atomic-like ground and first-excited electronic states centered on the radical metal atom. We theoretically investigate the extension of this idea to a larger scale by growing the alkyl group, $\mathrm{R}$, beyond the initial methyl group, $\mathrm{CH}_{3}$, while preserving the isolated and highly vertical character of the electronic excitation on the radical metal atom, M. Theory suggests that in the 
limit as the size of the ligand carbon chain increases, it can be considered a functionalized diamond (or cubic boron nitride) surface. Several requirements must be observed for the cycling centers to function when bound to the surface. First, the surface must have a significant band gap that fully encapsulates both the ground and excited states of the cycling center. Second, while the surface lattice imposes strict limits on the achievable spacing between the SrO- groups, at high coverage, SrO- centers can interact, and show geometric changes and/or electronic state mixing. We show that the coverage of the diamond surface with SrO- cycling centers needs to be significantly submonolayer for the functionality of the cycling center to be preserved. Having the lattice-imposed spatial control of SrO- placements will allow nanometer-scale proximity between qubits and will eliminate the need for atom traps for localized cycling emitters. Our results also imply that a functionalization could be done on a scanning microscope tip for local quantum sensing or on photonic structures for optically-mediated quantum information processing.

\section{Corresponding Author}

Corresponding Authors’ emails: jcaram@chem.ucla.edu, wes@physics.ucla.edu, ana@.chem.ucla.edu 


\section{Introduction}

Quantum information processing (QIP) is currently in a stage of parallel exploration of multiple platforms. Much like classical electronics, large-scale QIP will require approaches that are amenable to miniaturization and scalability. Along these lines, it seems fitting to approach building a quantum device (sensor, simulator, computer, etc.) by utilizing the natural scale at which quantum effects are important; for electrons at room temperature, this means the energy and size scale of single atoms. In contrast to larger systems that currently dominate the QIP landscape (such as superconducting qubits ${ }^{1}$ and phonons in trapped ion Coulomb crystals ${ }^{2}$, working at the nanometer scale has the potential advantage that inter-qubit coupling can be significantly stronger at short range, leading to systems that require less cooling to access pure collective states. However, single atom engineering at nanometer length scales is most naturally accomplished by chemically bonding the atoms to molecular hosts and using chemical (as opposed to mechanical) assembly. Chemical methods will therefore likely play a major role in development of quantum systems built from single molecular qubits.

Here, we present a new concept for molecular qubits that are designed to be scalable and packed at high density via surface modification, potentially produced by self-assembly of organic molecules. By attaching an optical cycling qubit moiety to a chemically defined crystal surface, we combine initialization and state readout based on optical cycling that trapped atoms provide, with the potential for the nanoscale lithographic control that surfaces furnish. In this way, this "chemically-trapped ion" platform borrows strengths from both gas-phase and solid state devices in an effort to build a concept that can leverage the best features of each for building the next generation of quantum devices. 


\section{Surface-Bound Optical Cycling Centers}

Recent successes in the laser cooling of molecules forms the basis of our design ${ }^{3-5}$ That work demonstrated that many photons can be spontaneously emitted by repeatedly laser-exciting optical transitions from polyatomic molecules without partitioning into excited molecular vibronic states. Common in these systems are 'functional groups' based on a metal atom that acts as optical cycling centers (OCCs) ${ }^{6}$ The primary feature of an OCC is an atom-centered optical electronic transition with near-zero Franck-Condon overlap for transitions that change its vibrational state. In this system, successive excitation and spontaneous emission from the OCC can result in a reduction of entropy as the internal state of the molecule is heralded, allowing for quantum state preparation (including laser cooling) and readout. The workhorse of this process is typically alkaline earth metal alkoxide groups (MO-R) in which a radical valence electron centered on the alkaline earth serves as the handle for state preparation and measurement (SPAM).

Recent calculations suggest that increasing the size and complexity of the ligands may not disrupt the closure of the optical cycle if the ligand is spatially and electronically separated from the OCC. ${ }^{6}$ This raises the possibility that an OCC can be chemically tethered to a substrate in vacuo while retaining its ability to spontaneously emit a series of photons upon state-selective excitation, providing readout of the initial internal quantum state of the molecule.

Beyond OCCs' roles in supplying an isolated transition in the molecule, they also provide a natural basis for storing and using quantum information. A quantum state stored in a pair of longlived sublevels of the electronic ground state (such as hyperfine structure) serves as a qubit and a state-selective optical cycle would allow excitation of only one of these qubit eigenstates (the "bright state") for laser-induced fluorescence, while the other eigenstate (the "dark state") would not emit fluorescence photons. Lack of collected fluorescence therefore constitutes detection (and 
heralds preparation) of the dark state, while detection of fluorescence indicates the bright state. As such, the optical cycling property allows SPAM of the molecular qubit, in a scheme analogous to those used in trapped ion quantum computers. ${ }^{7,8}$

Taken together, the tantalizing possibility that OCCs might be realized on surfaces and even patterned to produce multi-OCC interactions argues for a theoretical treatment of whether molecules hosting optical cycling centers can be attached to surfaces while retaining both chemical and physical properties. We approach this by expanding on the complexity of alkyl chain R-groups that are amenable to optical cycling. For strontium (Sr) as our example of an alkaline earth metal, we suggest that diamond and cubic boron nitride $(\mathrm{cBN})$ may serve as a good substrate for OCC anchoring and derive some general trends. We then explore how multiple OCCs may interact chemically or spectroscopically on a surface. We close by speculating on several schemes by which surface bound OCCs may provide next generation quantum systems through nanoscale control and patterning.

\section{Computational Methods}

We first probed the electronic structure of the smallest molecular emitters using MRCI/CASSCF (9 electrons/10 orbitals active space) with the def2-TZVPPD basis set. ${ }^{9-11}$ The geometries are optimized using the state-average CASSCF method (3 lowest-energy states with weights 0.8/0.1/0.1 for the ground state, and 0.2/0.4/0.4 for the first excited state given its neardegeneracy), and then the MRCI method is used to calculate electronic excitation energies with Molpro. ${ }^{12-17}$ These results were used to benchmark the performance of the Density Functional Theory (DFT) and Time-Dependent DFT (TD-DFT) methods ${ }^{18-21}$, implemented in the Gaussian 16 package ${ }^{22}$, capable of probing larger molecular systems. We show the comparison between DFT 
and MRCI/CASSCF for the geometries and excitation energies in Table S1. We found the PBE0 hybrid functiona ${ }^{23}$ with the $\mathrm{D} 3$ dispersion correction ${ }^{24}$ yielded the most reliable DFT-based results. It is additionally known to work well for excited state calculations. ${ }^{25-27} \mathrm{We}$ also probed the effects of basis set size and functional (Table S2). DFT geometry optimizations are performed with the def2-SVPD basis set, and a larger def2-TZVPPD basis set is used for excitation energy calculations. The effective core potential (ECP) is used for the $\mathrm{Sr}$ atom. The Franck-Condon Factors (FCFs) are calculated within the double-harmonic approximation using ezSpectrum ${ }^{28}$.

Geometry optimizations of OCCs bound to surfaces were performed using the Perdew-BurkeErnzerhof (PBE) functional, ${ }^{29,30}$ with the projector augmented-wave (PAW) potentials, ${ }^{31,32}$ implemented in the DFT-based Vienna $a b$ initio simulation package (VASP) ${ }^{31,33-36}$. The hybrid functional $\mathrm{HSE}^{37} \mathrm{6}^{37}$ is used for single point energy calculations, density of states calculations and the Bader charge analysis ${ }^{38,39}$. HSE06 has been extensively benchmarked against other functionals and performs well in predicting band gaps that fall within $1-5 \mathrm{eV} \cdot .^{40,41} \mathrm{~A} 1 \times 1 \times 1$ MonkhorstPack $k$-point grid centered at $\Gamma$-point and a plane-wave cutoff energy of $400 \mathrm{eV}$ are employed, with a convergence criterion of $10^{-6} \mathrm{eV}$ for the SCF relaxation. The $k$-point grid is considered sufficient due to the large size of the unit cell and the significant separation between OCCs that we probe, expected to produce flat bands, accurately modeled with just the $\Gamma$-point calculation. A five-layer $2 \times 2$ supercell with the exposed (111) facet, in periodic boundary conditions, represents the cubic diamond surface as an infinite slab, in which the two surfaces are covered with $\mathrm{H}$ atoms. In this supercell $160 \mathrm{C}$ atoms in total, of which 64 are surface-exposed (or 32 per face), all capped with $\mathrm{H}$ before $\mathrm{SrO}$ deposition. The dimensions of the supercell are $14 \AA \times 14 \AA \times 20 \AA$, which we found to be sufficient to prevent the interaction between the periodic images. The top-three layers 
of $\mathrm{C}$ atoms and the surface $\mathrm{H}$ atoms are relaxed during the geometry optimizations. The optimizations are considered converged when the residual forces are less than $0.01 \mathrm{eV} /$ atom.

\section{Results and Discussion}

\section{Molecular alkoxide radicals}

The benchmarking of the computational methodology was done on the $\mathrm{SrOH}$ species, for which the experimental data is available, and high-level ab initio theory is affordable. TD-DFT results agree well with the MRCI/CASSCF results and the experiment ${ }^{42,43}$ (Tables S1, S2). The TD-DFT optimized Sr-O bond lengths deviate from the experiment values by less than $0.02 \AA$, and the differences between TD-DFT and CASSCF are less than $0.06 \AA$. More importantly, for both TDDFT and CASSCF methods, the computed bond length differences between the ground and excited states differ from the experimental data by $0.002-0.008 \AA$. All considered functionals give reasonable results for $\mathrm{Sr}-\mathrm{O}$ bond length and bond length change. The TD-DFT excitation energy agrees with experimental results within $0.1 \mathrm{eV}$, and the PBE0 hybrid functional performs best in this regard. We also probed the effect of functionals and basis sets. In order to further reduce the computation cost, we use a smaller basis set, def2-SVPD, for geometry optimization and a larger basis set, def2-TZVPPD, for excitation energy calculations. Table S2 shows that this strategy gives a good agreement for bond length change and excitation energy with the results from the def2TZVPPD basis set. Thus, TD-DFT should provide accurate descriptions for alkoxide radicals. The good performance of DFT is not unexpected, because for optimized OCC the wavefunctions on both electronic states are single configuration and located far from crossings with other electronic states. 
a
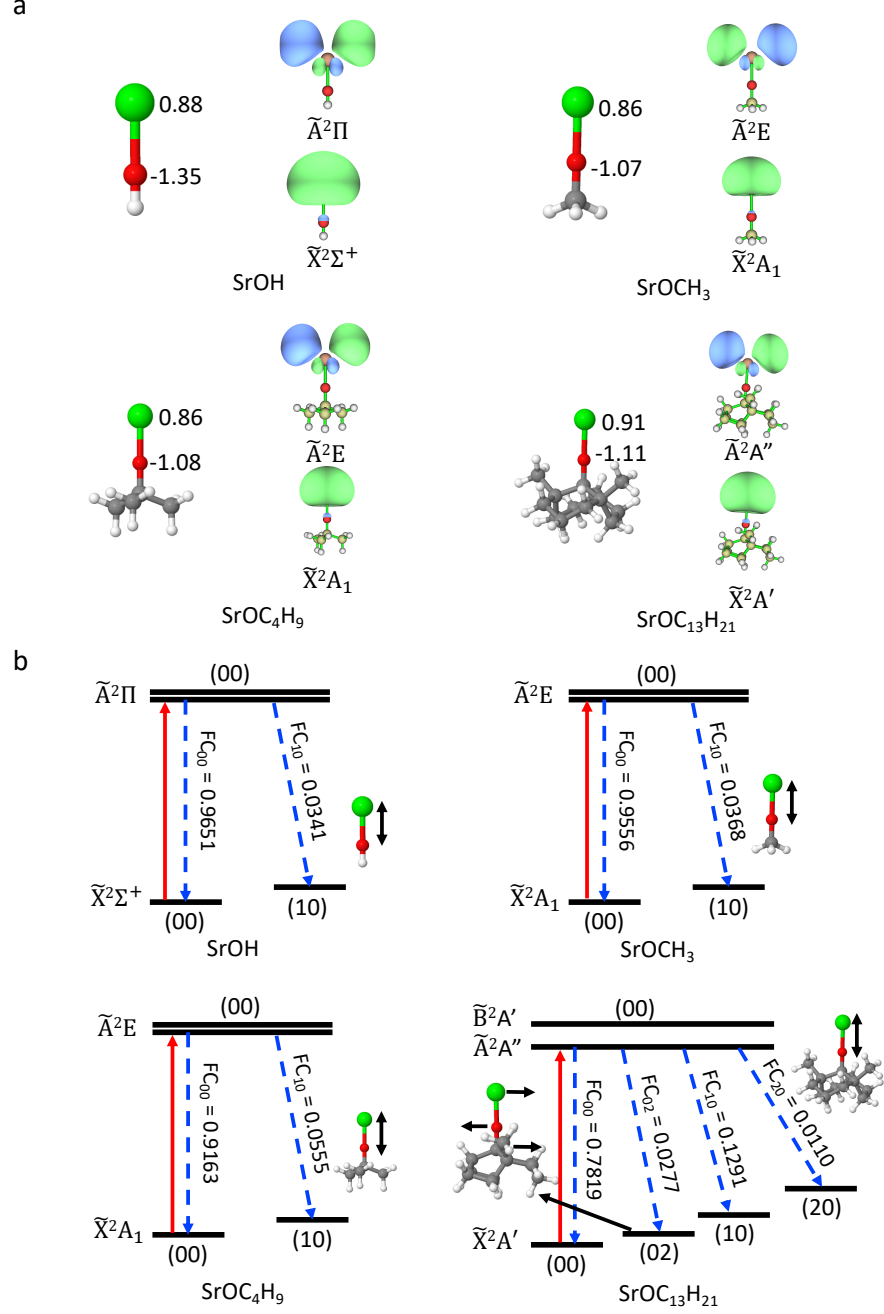

Figure 1. (a) Optimized ground-state geometries of SrOR. Atomic charges on the Sr and O atoms, computed from NPA, are also shown in the left side of each sub-figure. The right side of each subfigure shows the NTO for Highest Occupied Molecular Orbitals (HOMO) and Lowest Unoccupied Molecular Orbitals (LUMO). The isosurfaces are plotted with an isovalue of 0.03. (b) Photon cycling scheme for SrOR. Excitation from the ground state to the first excited state and spontaneous decay channels are shown with upward red arrows and downward blue dashed arrows. The FCFs are shown along with each decay. The vibrational quantum numbers $\left(v_{1} v_{2}\right)$ correspond to the $\mathrm{Sr}-\mathrm{O}$ stretching $\left(v_{1}\right)$ and $\mathrm{Sr}-\mathrm{O}-\mathrm{C}$ bending $\left(v_{2}\right)$ modes. 
Table 1. Electronic excitation properties for SrOR. $\Delta \mathrm{r}_{\mathrm{Sr}-\mathrm{O}}$ is the $\mathrm{Sr}-\mathrm{O}$ bond length change from the ground state to the first excited state $\left(\Delta \mathrm{r}_{\mathrm{Sr}-\mathrm{O}}=\mathrm{r}_{\mathrm{Sr}-\mathrm{O}}^{\mathrm{ex}}-\mathrm{r}_{\mathrm{Sr}-\mathrm{O}}^{\mathrm{gs}}\right), \Delta E$ is the computed vertical excitation energy, and FCF is the Franck Condon Factor for the decay transition from the first excited state to the ground state. The initial and target states are vibrational ground states.

\begin{tabular}{|l|l|l|l|}
\hline Molecule & $\Delta \mathrm{r}_{\mathrm{Sr}-\mathrm{O}}(\AA)$ & $\Delta E(\mathrm{eV})$ & FCF \\
\hline $\mathrm{SrOH}$ & -0.017 & 1.750 & 0.9651 \\
\hline $\mathrm{SrOCH}_{3}$ & -0.018 & 1.754 & 0.9556 \\
\hline $\mathrm{SrOC}_{4} \mathrm{H}_{9}$ & -0.021 & 1.738 & 0.9163 \\
\hline $\mathrm{SrOC}_{13} \mathrm{H}_{21}$ & -0.029 & 1.721 & 0.7819 \\
\hline
\end{tabular}

We start from the hypothesis that the alkyl group R, in SrOR, can be increased beyond methyl, $\mathrm{CH}_{3}$. We apply the TD-DFT method to a series of SrOR molecular radicals, $\mathrm{R}$, with increasing numbers of carbon atoms, $\mathrm{CH}_{3}, \mathrm{C}_{4} \mathrm{H}_{9}, \mathrm{C}_{13} \mathrm{H}_{21}$ (Figure 1). Our goal is to progressively build up to the local structure of a diamond surface. We focus on the changes in geometries within the $\mathrm{SrO}$ part of the molecular radicals in the ground and first excited states, the excitation energies, and the Frank-Condon factors, FCFs. All the cluster models can maintain a local linear structure, with the Sr-O-C angles of $179.8-179.9^{\circ}$ for the ground state, and $179.6-180.0^{\circ}$ for the excited state. This bond angle is characteristic also of $\mathrm{SrOH}$, and suggests a strongly ionic bond character between $\mathrm{Sr}$ and the $\mathrm{O}$ atom. As shown in Table 1, the two states in these models are aligned within $0.02-0.03$ 
$\AA$ in terms of the equilibrium Sr-O bond length, and larger R sizes correspond to slight deviations from the linear character.

The three cluster models exhibit similar charge distribution on the $\mathrm{Sr}$ and $\mathrm{O}$ atoms from the natural population analysis (NPA) ${ }^{44}$, due to the similar electron withdrawing strength of the alkoxide groups bound to Sr. The natural transition orbitals (NTOs) in all cluster-bound SrO have the same shape as those in $\mathrm{SrOH}$, with electron density localized on the $\mathrm{Sr}$ atom (Figure 1). This suggests that the perturbation from the R group is minor. However, we observe that, as the cluster size increases, a small amount of electron density appears on the $\mathrm{O}$ atom and some on the $\mathrm{C}$ atoms, which will make the potential energy surfaces (PESs) of the ground $(\tilde{X})$ and first excited $(\tilde{A})$ states, less parallel, and thus the FCF less diagonal (deviating more form the highly diagonal FCF in $\left.\mathrm{SrOH}^{5,6}\right)$. This is because more modes are contributing to the off-diagonal FCFs. In addition, the contribution from the Sr-O stretching mode is slightly increased, consistent with our observation that the Sr-O bond length change is increased, and thus the PESs of the two states are less parallel along this mode as we move to larger model systems. However, the decrease in FCF, due to the size effect, is not significant, and $\mathrm{SrO}$ will still have a reasonably good FCF. Note as well that the diamond-supported system will have a very different reduced mass.

In $\mathrm{SrOC}_{13} \mathrm{H}_{21}$, there is a very small amount of electron density on the two $\mathrm{C}$ atoms that are not directly bound to $\mathrm{O}$, but are only $2.7 \AA$ apart from the $\mathrm{O}$ atom. These carbon atoms will conform to the top-layer atoms on the diamond surface, which are $2.9 \AA$ away from $\mathrm{O}$ in Figure 2. Consistently, we find that the distance between the $\mathrm{O}$ atom and these $\mathrm{C}$ atoms changes by $0.004 \AA$ from the $\tilde{X}^{2} A^{\prime}$ to the $\tilde{A}^{2} A^{\prime \prime}$ state, and that constitutes the second largest geometric changes in $\mathrm{SrOC}_{13} \mathrm{H}_{21}$. Therefore, when the cycling center is deposited onto the diamond surface, the orbitals of these two $\mathrm{C}$ atoms may have weak couplings to the $\mathrm{O}$ and $\mathrm{Sr}$ atoms and affect the FCF. On the 
other hand, the chemical nature of these atoms in the diamond surface is not identical to that in a finite hydrocarbon, and thus, this small involvement of the $\mathrm{C}$ atoms of the $\mathrm{C}_{13} \mathrm{H}_{21}$ system in the key transition does not necessarily preclude the possibility of the infinite surface to be a viable OCC platform.

The TD-DFT calculations show that the first vertical excitation energy is slightly decreased as the size of the $\mathrm{R}$ group increases (Table 1), agreeing well with previous experimental measurements $^{42}$ (Table S3). Given this trend, the diamond-bound $\mathrm{SrO}$ is expected to have slightly lower excitation energy than $\mathrm{SrOH}$.

\section{Surface functionalization with SrO cycling centers}

Encouraged by the FCFs observed so far in small clusters, we now expand the R group to encompass the full diamond surface. We first explore the ground state structure of SrO bound to diamond, and compare those to the gas-phase $\mathrm{SrOH}$ radical, which is known to have a diagonal FCF. The Sr-Sr separation afforded within this supercell is $14.27 \AA$. When the $\mathrm{SrO}$ group is bound to the surface, the Sr-O-C unit can maintain a roughly linear geometry (Figure 2), with an angle of $175.2^{\circ}$. The ground-state $\mathrm{Sr}-\mathrm{O}$ bond length is $2.125 \AA$ on the diamond surface, i.e. $0.019 \AA$ longer than that of the gas-phase $\mathrm{SrOH}$ molecule. The atomic Bader charge on Sr within the surfacebound $\mathrm{SrO}$ (Figure 2 (a) and (b)) is +0.85 e, i.e. similar to that in $\mathrm{SrOH}$ and nearly identical to those seen in $\mathrm{SrOCH}_{3}$ and $\mathrm{SrOC}_{4} \mathrm{H}_{9}$ (Figure 1), indicating an unpaired electron localized on the cycling center. The overall resemblance of the surface-bound $\mathrm{SrO}$ to the best cycling molecules suggests that optical efficient cycling should still be possible at this limit.

Figure 2 (c, d) show the electron density of the highest occupied molecular orbital (HOMO), occupied by the unpaired electron, and the doubly-degenerate lowest unoccupied molecular orbital 
(LUMO) of the $\mathrm{SrOH}$ molecule and the $\mathrm{SrO}$ group attached to diamond. For both HOMO and LUMO of surface-bound SrO, a majority of electron density is localized on Sr, leading to electronic transition localized on the cycling center. Again, the molecular orbitals of the surfacebound $\mathrm{SrO}$ have similar shapes to those of the $\mathrm{SrOH}$ radical, suggesting similar distributions of atomic orbital characters. For $\mathrm{SrOH}$ in the $\tilde{X}^{2} \Sigma^{+}$state, the orbital occupied by the unpaired electron is dominated by the $\operatorname{Sr} s$ character with the remaining contribution from the $\operatorname{Sr} p$ orbital, whereas for the $\tilde{A}^{2} \Pi$ state, the $\operatorname{Sr} p$ and $d$ orbitals dominate the molecular orbital character.

a

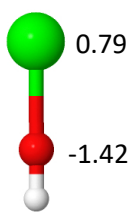

C<smiles>C1=C2CCCC23CCCCC3CC1</smiles>

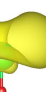

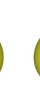

LUMO

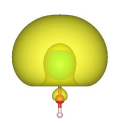

HOMO

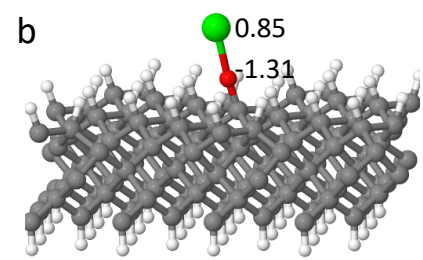

d

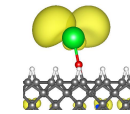

LUMO

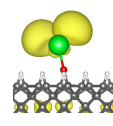

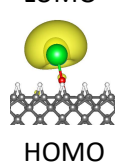

e

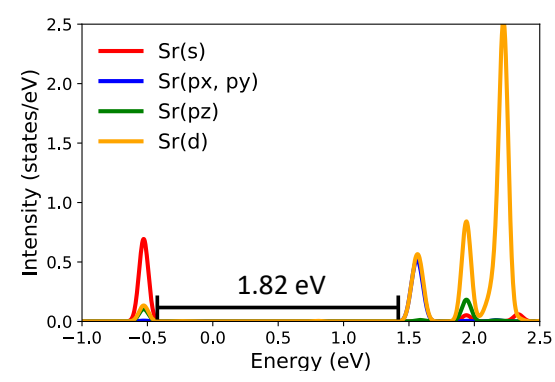

Figure 2. (a) (b) Optimized geometries of the gas-phase $\mathrm{SrOH}$ molecule and the SrO-group bound to the diamond surface. The green, red, gray and white colors represent the $\mathrm{Sr}, \mathrm{O}, \mathrm{C}$ and $\mathrm{H}$ atoms, respectively. The atomic charges on $\mathrm{Sr}$ and $\mathrm{O}$ from the Bader charge analysis are also shown. (c) (d) Electron density of the HOMO and LUMO of the $\mathrm{SrOH}$ molecule and the $\mathrm{SrO}$ supported on 
diamond. The isosurfaces are plotted with an isovalue of 0.001. (e) PDOS, indicating the excitation energy distance.

Given the similarity in geometry, charge distribution and shapes of the HOMO and LUMO between the diamond-surface-bound $\mathrm{SrO}$ and the $\mathrm{SrOH}$ molecule, we expect $\mathrm{SrO}$ to maintain its excitation properties once bound to the diamond surface. We can roughly estimate the excitation energy from the projected density of states (PDOS) (Figure 2e), to be $1.82 \mathrm{eV}$ for diamond-bound SrO. This is close to the excitation energy in $\mathrm{SrOH}(1.83 \mathrm{eV})$, estimated from the PDOS calculation. While the number derived from PDOS is necessarily approximate, the HSE06 functional is known to be quite reliable for the optical gap, as discussed above. Calculating the FCF for the infinite surface is unfortunately technically impossible at the moment, and thus, our results are a proposal, substantiated as best as currently possible. Confirming this obviously requires experimental validation.

Next, we believe there is a rational way to improve the properties of the surface-bound $\mathrm{SrO}$, through changing the support to a close diamond analogue, cubic boron nitride (cBN). $\mathrm{cBN}$ has the same number of valence electrons and the same crystal structure as the cubic diamond, and thus, mirrors diamond's properties. However, it additionally features a small amount of charge transfer between B and N. Given that the surface of $\mathrm{cBN}$ is expected to be B-terminated, and that SrO- would therefore bind to the boron, the intra-crystal polarization is expected to effectively create a more electron-withdrawing ligand, R, for SrO-. Figure 3 shows the optimized structure for SrO-cBN from periodic DFT calculations. The Sr-O bond is $2.104 \AA$ on cBN, i.e. $0.021 \AA$ shorter than that on diamond. The Sr-O-B can maintain a linear structure near Sr, with a nowperfect angle of $180.0^{\circ}$. In line with this finding, and our hypothesis regarding $\mathrm{cBN}$, the $\mathrm{Sr}$ atom has more positive charge on $\mathrm{cBN}$ than that on diamond. The HOMO and LUMO of this system 
(Figure 3) are the expected singly-occupied states, very similar to those of SrO- on diamond and $\mathrm{SrOH}$ in the gas phase. From the PDOS, the excitation energy of SrO- on cBN is $1.70 \mathrm{eV}$. Thus, we see that the behavior of OCC is likely again preserved on $\mathrm{cBN}$ at this low coverage. While the experimental confirmation of the OCC behavior on both surfaces is warranted, our results suggest that both diamond and $\mathrm{cBN}$ could be used to support the $\mathrm{SrO}-$ cycling radical centers, and $\mathrm{cBN}$ has a potential to be a superior support.

a

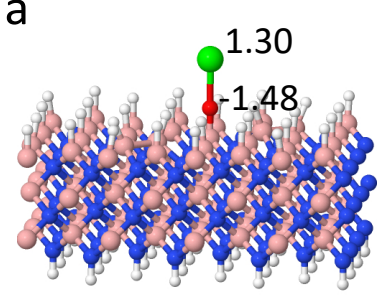

b

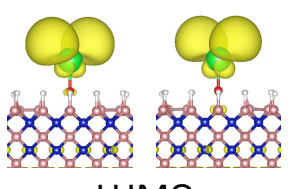

LUMO

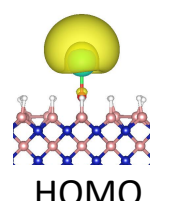

C

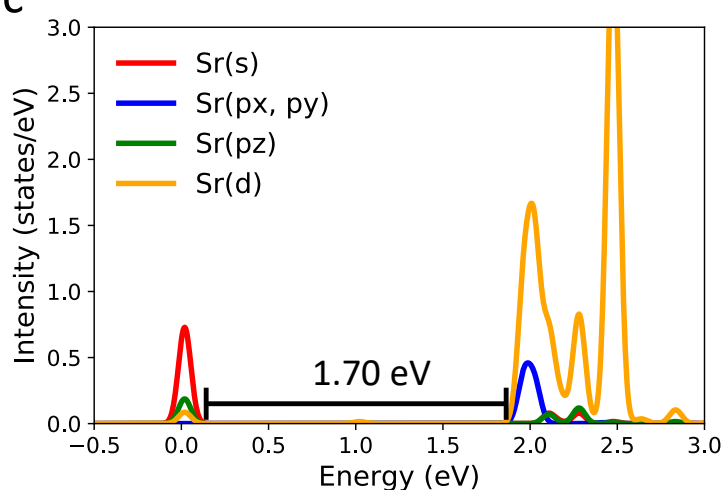

Figure 3. (a) Optimized geometry of the SrO- group bound to the boron nitride surface. The green, red, pink, blue and white colors represent the $\mathrm{Sr}, \mathrm{O}, \mathrm{B}, \mathrm{N}$ and $\mathrm{H}$ atoms, respectively. The atomic charges on $\mathrm{Sr}$ and $\mathrm{O}$ from the Bader charge analysis are also shown. (b) Electron density of the HOMO and the LUMO of the SrO- supported on cBN. The isosurfaces are plotted with an isovalue of 0.001. (c) PDOS, indicating the excitation energy distance. 


\section{Effect of surface coverage}

Finally, the interactions and entanglement between the optical cycling centers would be affected by their spatial separation. The surface allows for certain control of it, since the affordable separations are multiples of the surface lattice constant. We explore the effect of surface coverage on the properties of the cycling centers. First, we have studied the interactions between the SrOgroups when they are uniformly distributed on diamond, by changing the size of the periodic supercell. The structure in Figure 2 corresponds to a coverage of 1/32 monolayer (ML), and it was chosen specifically to avoid the interactions between the Sr centers in the neighboring unit cells. Below we address what happens if coverage increases to $1 / 8$ or even $1 / 2 \mathrm{ML}$.

a

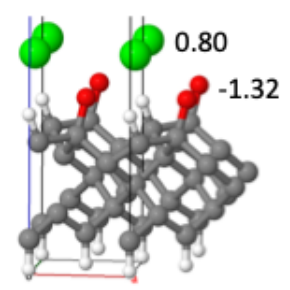

$1 / 2$ coverage

c

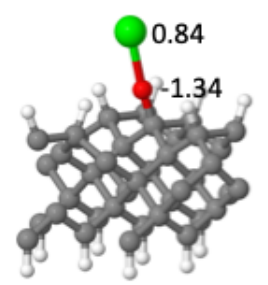

$1 / 8$ coverage

b

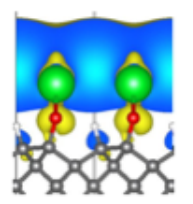

Hомо

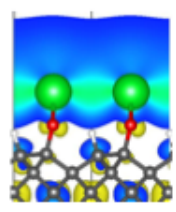

LUMO

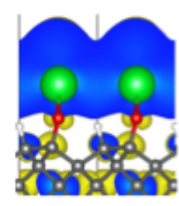

LUMO+1

d

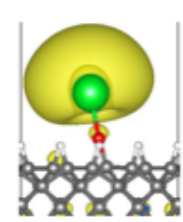

HOMO

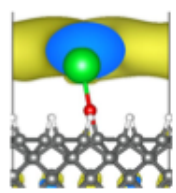

LUMO

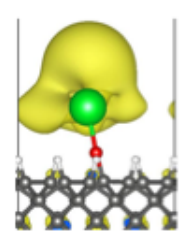

LUMO+1

Figure 4. (a) (c) Optimized geometries for SrO-diamond with the cycling center coverage of $1 / 2$ and 1/8. Two unit cells are shown for the case of 1/2 coverage. (b) (d) Electron density of the HOMO, LUMO, and LUMO+1 for different coverages of cycling centers. The isosurfaces are plotted with an isovalue of 0.001 . 
When the cycling center coverage is $1 / 2 \mathrm{ML}$, Sr will bind to two $\mathrm{O}$ atoms, with bond lengths of $2.33 \AA$ and $2.39 \AA$, respectively (Figure 4). The distance between the two Sr centers is $3.57 \AA$. The HOMO and LUMO are completely delocalized, where the adjacent $\mathrm{Sr}$ radical orbitals mix, effectively forming a half-filled $\sigma$-band. When the coverage is $1 / 8 \mathrm{ML}$, corresponding to the distance between the two cycling centers of $7.13 \AA$, the $\mathrm{Sr}-\mathrm{O}-\mathrm{C}$ unit can maintain the locally linear structure. However, the orbitals of two neighboring Sr atoms still interact with each other, either polarizing the original orbitals, or mixing and splitting into two mixed states.

a

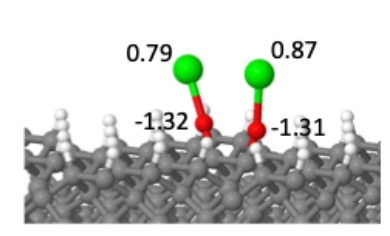

b

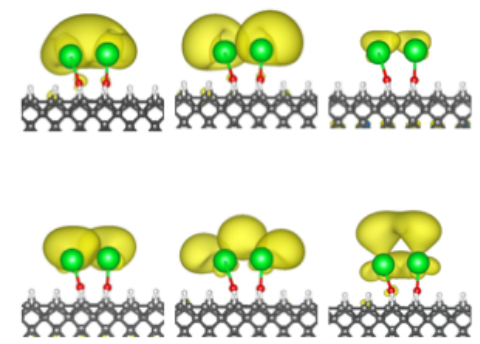

Figure 5. (a) Optimized geometry for two cycling centers on the diamond surface. The atomic charges on $\mathrm{Sr}$ and $\mathrm{O}$ from the Bader charge analysis are also shown. (b) Electron density for 6 low energy orbitals. The isosurfaces are plotted with an isovalue of 0.001 .

We have also studied the systems where two SrO- units are in the same unit cell, and can form dimer geometries. In some cases, one $\mathrm{Sr}$ will bind to two $\mathrm{O}$ atoms, similar to that in Figure 4 (a), while in other cases the two SrO- groups can maintain the locally linear structures, but their orbitals interact with each other. Figure 5 shows one case where the distance between two $\mathrm{Sr}$ is $3.73 \AA$, and the distance between the two $\mathrm{C}$ atoms that the $\mathrm{SrO}$ - groups are bound to is $1.71 \AA$. The two $\mathrm{Sr}$ $s$ orbitals with the same energy form a bonding and an antibonding combinations, while the doubly 
degenerate $p$ - and $d$ - type orbitals from different $\mathrm{Sr}$ form even more mixed orbitals. The charges on the Sr atoms also suggest that they are not chemically identical.

Clearly, these coverages are too high to maintain the clean radical character of the cycling centers in the ground and excited states. Our results indicate that the surface coverage with radical center has to be certainly lower than $1 / 8 \mathrm{ML}$, in order for the cycling centers to maintain their local electronic character and the diagonal FCF.

\section{Conclusions and Future Prospects}

In this contribution we showed that the $\mathrm{SrO}$ - radical cycling center can be attached to $\mathrm{R}$ groups as large as the diamond or cBN surface. Surface patterning with such centers allows for spatial control and alignment. The coverage of the surface with the cycling centers cannot be very high, to prevent their chemical interaction with each other (as seen at $1 / 2 \mathrm{ML}$ ), or mutual electronic perturbation (as seen at 1/8 ML). Even though at 1/8 ML the geometries of the SrO- units are linear, their electronic states can still mix, particularly on the excited state. Furthermore, our results suggest that the surfaces that can successfully host radical cycling centers must feature large band gaps, such that the HOMO and LUMO of the radical are located safely within the gap and not mix with the states of the surface.

We hypothesize that surface supported optical cycling ligands can be realized through chemical functionalization of the surface. We propose that diamond grown by chemical vapor deposition (CVD) (which is typically H-terminated) can be functionalized with pendant hydroxide groups by low-pressure oxygen plasma treatment ${ }^{48,49}$. Following this, reactive $\mathrm{Sr}$ can be prepared by exciting the $\mathrm{Sr}$ atoms to the metastable state ${ }^{3} \mathrm{P}_{1}$ with laser excitation ${ }^{50-52}$. To control the deposition, the 
amount of pendant hydroxide can be tuned by modulating the power and duration of the plasma treatment.

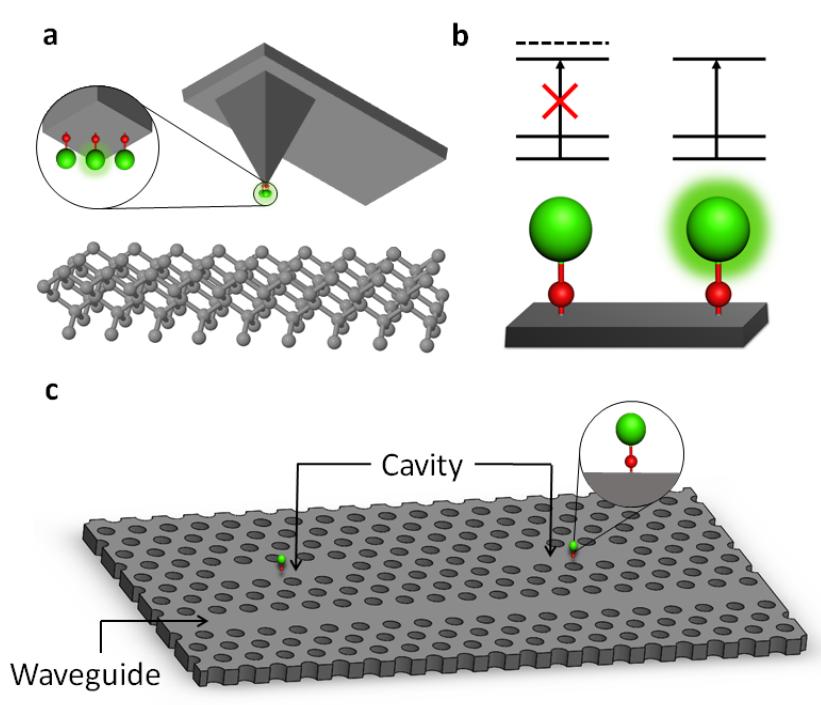

Figure 6. Applications of surface bound OCCs. (a) An AFM/STM tip can be functionalized with OCC centers, enabling precision quantum-enhance readout of local magnetic and electric fields through fluorescence. (b) Two OCCs in spatial proximity can provide the two qubit basis for a CNOT gate achieved through dipole blockade. (c) OCCs can be placed in a photonic waveguide, entangling two OCCs via photonic bus.

Figure 6 shows three applications that would be enabled by the development of surface-bound, state-selective emitters. For sensing applications, Figure 6 (a) shows a conceptual arrangement whereby a single molecular qubit could be used for spatial imaging of, for instance, local magnetic fields by functionalizing the tip of a scanning tunneling microscope (STM) or an atomic force microscope (AFM). Light for SPAM can be delivered to and collected from the OCC via fibers or optical waveguides built into the tip. A superposition of field-sensitive qubit states will process at a rate determined by the local magnetic field, and readout after free precession can be used to map the field at the atomic scale. 
Note, however, that, if AFM is conducted at ambient pressures, the $\mathrm{Sr}$ radicals are likely to chemically react with the gases, and quench. For STM, the tip generates an oriented electric field, which at large enough magnitudes may affect charge separation and hinder Franck-Condon factors. Experimentally, an external electric field generated by STM was shown to stabilize or destabilize charge-transfer in organic molecules placed on gold surfaces, based on alignment. The external electric field strengths studied ranged from $-1 \times 10^{9}$ to $1 \times 10^{9} \mathrm{~V} / \mathrm{m} .{ }^{53}$

To probe how external fields would affect optical cycling, we introduced electrical fields in two ways. A field between $-1 \times 10^{10}$ to $1 \times 10^{10} \mathrm{~V} / \mathrm{m}$ aligned with the $\mathrm{Sr}-\mathrm{O}$ bond was applied to $1 / 32 \mathrm{ML}$ Sr-O OCCs on diamond (Table S5). In another case, a field of $1 \times 10^{8}-1 \times 10^{10} \mathrm{~V} / \mathrm{m}$ was applied to two SrO- units located $3.88 \AA$ apart and 10.39 ̊̊ from other OCCs on diamond (Table S6). In both cases, larger electric fields reduced charge localization on $\mathrm{Sr}$, while the $\mathrm{Sr}-\mathrm{O}$ bond length would increase or decrease depending on the direction of the field. The effect on charge can be explained by the fact that the field pushes the full electron density in the system, affecting the bonding character on the ground and excited state beyond just the radical-centered HOMO and LUMO. We made a comparison of these results to the cluster model, $\mathrm{SrOCH}_{3}$, with an external electric field applied along the Sr-O bond. As on the surface, the applied field led to charge delocalization and increase or decrease in Sr-O bond length, depending on the field direction. In both field directions, the ground versus excited state geometry difference increases with increasing field magnitude, implying poorer FCFs (Table S7). Thus, in STM, large electric fields can adversely affect radical charge localization and FCF of OCCs.

In Figure 6 (b), two qubits in a quantum processor are shown performing a controlled-NOT (CNOT) quantum gate. Here, the proximity of the qubits can be extremely close (but not closer than $1 / 32$ ML on diamond, in our calculations), boosting both the energy scale and speed of the 
gate. A transition coupled to only one of the two qubit eigenstates can be used to create a dipole blockade $^{54}$, a paradigm in which the dipole-dipole interaction is strong enough to shift the doubleexcitation energy more than the transition linewidth. As such, precisely one of the two qubits can be excited, and an initially separable system can be left in a well-defined entangled state. Along with a complete set of single-molecule operations, this two-qubit interaction is sufficient for universal quantum computation.

As a third example, Figure 6 (c) shows a photonic interconnect for large-scale processing based on surface-bound OCCs. Even qubits that are physically distant can interact strongly with one another due to their strong coupling to photonic cavities built from their substrates, and classical routers can turn interactions between individual qubits on and off by optically connecting their photonic cavities together.

We examine the possibility of constructing pendant radical alkaline earth based OCCs on surfaces. Such structures may bridge surface engineering with the precise quantum control afforded by atomic physics. After validating our computational methods, we find that diamond and cBN could support SrO- based OCCs. We establish the necessary density of OCCs on a surface needed to retain independent electronic character and explore how they might be used in applications such as STMs, universal quantum gates, or as substrates for quantum photonics. We believe this presents a path forward to realizing a novel quantum architecture experimentally.

\section{Author Contributions}

HG, ANA, WC, JRC generated the main project idea. HG and CD performed all calculations. ANA supervised the technical execution of the project. All Authors discussed and analyzed the 
results. The manuscript was written through contributions of all authors. All authors have given approval to the final version of the manuscript.

\section{Funding Sources}

Any funds used to support the research of the manuscript should be placed here (per journal style).

\section{ACKNOWLEDGMENT}

This work was supported by the Department of Energy Basic Energy Sciences grant DESC0019152. This work used computational and storage services associated with the Hoffman2 cluster provided by the UCLA Institute for Digital Research and Education's Research Technology Group, the NSF-supported Extreme Science and Engineering Discovery Environment (XSEDE) (TG-CHE160054). 


\section{REFERENCES}

(1) Krantz, P.; Kjaergaard, M.; Yan, F.; Orlando, T. P.; Gustavsson, S.; Oliver, W. D. Appl. Phys. Rev. 2019, 6, 021318.

(2) Bruzewicz, C. D.; Chiaverini, J.; McConnell, R.; Sage, J. M. Appl. Phys. Rev. 2019, 6, 021314.

(3) Shuman, E. S.; Barry, J. F.; Glenn, D. R.; Demille, D. Phys. Rev. Lett. 2009, 103 (22), 223001.

(4) Hummon, M. T.; Yeo, M.; Stuhl, B. K.; Collopy, A. L.; Xia, Y.; Ye, J. Phys. Rev. Lett. 2013, 110 (14), 143001.

(5) Kozyryev, I.; Baum, L.; Matsuda, K.; Augenbraun, B. L.; Anderegg, L.; Sedlack, A. P.; Doyle, J. M. Phys. Rev. Lett. 2017, 118 (17), 173201(6).

(6) Kozyryev, I.; Baum, L.; Matsuda, K.; Doyle, J. M. ChemPhysChem 2016, 17 (22), 36413648 .

(7) Myerson, A. H.; Szwer, D. J.; Webster, S. C.; Allcock, D. T. C.; Curtis, M. J.; Imreh, G.; Sherman, J. A.; Stacey, D. N.; Steane, A. M.; Lucas, D. M. Phys.Rev. Lett. 2008, 100 (20), 200502.

(8) Blatt, R.; Roos, C. F. Nat. Phys. 2012, 8 (4), 277-284.

(9) Pritchard, B. P.; Altarawy, D.; Didier, B.; Gibson, T. D.; Windus, T. L. J. Chem. Inf. Model. 2019, $59(11), 4814-4820$.

(10) Weigend, F. Phys. Chem. Chem. Phys. 2006, 8 (9), 1057-1065. 
(11) Weigend, F.; Ahlrichs, R. Phys. Chem. Chem. Phys. 2005, 7 (18), 3297-3305.

(12) Werner, H. J.; Knowles, P. J.; Knizia, G.; Manby, F. R.; Schütz, M. Wiley Interdiscip. Rev. Comput. Mol.Sci. 2012, 2 (2), 242-253.

(13) Knowles, P. J.; Werner, H. J. Theor. Chim. Acta 1992, 84, 95-103.

(14) Knowles, P. J.; Werner, H. J. Chem. Phys. Lett. 1988, 145 (6), 514-522.

(15) Werner, H. J.; Knowles, P. J. J. Chem. Phys. 1988, 89 (9), 5803-5814.

(16) Werner, H. J.; Knowles, P. J. J. Chem. Phys. 1985, 82 (11), 5053-5063.

(17) Werner, H.-J.; Knowles, P. J.; Knizia, G.; Manby, F. R.; Schütz, M.; Celani, P.; Györffy, W.; Kats, D.; Korona, T.; Lindh, R.; Mitrushenkov, A.; Rauhut, G.; Shamasundar, K. R.; Adler, T. B.; Amos, R. D.; Bennie, S. J.; Bernhardsson, A.; Berning, A.; Cooper, D. L.; Deegan, M. J. O.; Dobbyn, A. J.; Eckert, F.; Goll, E.; Hampel, C.; Hesselmann, A.; Hetzer, G.; Hrenar, T.; Jansen, G.; Köppl, C.; Lee, S. J. R.; Liu, Y.; Lloyd, A. W.; Ma, Q.; Mata, R. A.; May, A. J.; McNicholas, S. J.; Meyer, W.; \{Miller III\}, T. F.; Mura, M. E.; Nicklaß, A.; O’Neill, D. P.; Palmieri, P.; Peng, D.; Pflüger, K.; Pitzer, R.; Reiher, M.; Shiozaki, T.; Stoll, H.; Stone, A. J.; Tarroni, R.; Thorsteinsson, T.; Wang, M.; Welborn, M. .

(18) Liu, J.; Liang, W. J. Chem. Phys. 2011, 135 (18), 184111.

(19) Liu, J.; Liang, W. J. Chem. Phys. 2011, 135 (1), 014113.

(20) Furche, F.; Ahlrichs, R. J. Chem. Phys. 2002, 117 (16), 7433-7447.

(21) Bauernschmitt, R.; Ahlrichs, R. Chem. Phys. Lett. 1996, 256, 454-464. 
(22) Frisch, M. J.; Trucks, G. W.; Schlegel, H. B.; Scuseria, G. E.; Robb, M. A.; Cheeseman, J. R.; Scalmani, G.; Barone, V.; Mennucci, B.; Petersson, G. A.; Nakatsuji, H.; Caricato, M.; Li, X.; Hratchian, H. P.; Izmaylov, A. F.; Bloino, J.; Zheng, G.; Sonnenberg, J. L.; Hada, M.; Ehara, M.; Toyota, K.; Fukuda, R.; Hasegawa, J.; Ishida, M.; Nakajima, T.; Honda, Y.; Kitao, O.; Nakai, H.; Vreven, T.; Montgomery, J. A., J.; Peralta, J. E.; Ogliaro, F.; Bearpark, M.; Heyd, J. J.; Brothers, E.; Kudin, K. N.; Staroverov, V. N.; Kobayashi, R.; Normand, J.; Raghavachari, K.; Rendell, A.; Burant, J. C.; Iyengar, S. S.; Tomasi, J.; Cossi, M.; Rega, N.; Millam, J. M.; Klene, M.; Knox, J. E.; Cross, J. B.; Bakken, V.; Adamo, C.; Jaramillo, J.; Gomperts, R.; Stratmann, R. E.; Yazyev, O.; Austin, A. J.; Cammi, R.; Pomelli, C.; Ochterski, J. W.; Martin, R. L.; Morokuma, K.; Zakrzewski, V. G.; Voth, G. A.; Salvador, P.; Dannenberg, J. J.; Dapprich, S.; Daniels, A. D.; Farkas, Ö.; Foresman, J. B.; Ortiz, J. V.; Cioslowski, J.; Fox, D. J. Gaussian, Inc., Wallingford CT 2009.

(23) Perdew, J. P.; Ernzerhof, M.; Burke, K. J. Chem. Phys. 1996, 105 (22), 9982-9985.

(24) Grimme, S.; Antony, J.; Ehrlich, S.; Krieg, H. J. Chem. Phys. 2010, 132 (15), 154104.

(25) Shao, Y.; Mei, Y.; Sundholm, D.; Kaila, V. R. I. J. Chem. Theory Comput. 2020, 16, 587600.

(26) Robinson, P. J.; Zhang, X.; McQueen, T.; Bowen, K. H.; Alexandrova, A. N. J. Phys. Chem. A 2017, 121 (8), 1849-1854.

(27) Adamo, C.; Barone, V. J. Chem. Phys. 1999, 110 (13), 6158-6170.

(28) Mozhayskiy, V. A.; Krylov, A. I. ezSpectrum.

(29) Perdew, J. P.; Burke, K.; Ernzerhof, M. Phys. Rev. Lett. 1997, 78 (7), 1396-1396. 
(30) Perdew, J. P.; Burke, K.; Ernzerhof, M. Phys. Rev. Lett. 1996, 77 (18), 3865-3868.

(31) Kresse, G.; Joubert, D. Phys. Rev. B 1999, 59 (3), 1758-1775.

(32) Blöchl, P. E. Phys. Rev. B 1994, 50 (24), 17953-17979.

(33) Kresse, G.; Furthmüller, J. Phys. Rev. B Condens. Matter Mater. Phys. 1996, 54 (16), 11169-11186.

(34) Kresse, G.; Furthmüller, J. Comput. Mater. Sci. 1996, 6 (1), 15-50.

(35) Kresse, G.; Hafner, J. Phys. Rev. B Condens. Matter Mater. Phys. 1994, 49 (20), 1425114269.

(36) Kresse, G.; Hafner, J. Phys. Rev. B Condens. Matter Mater. Phys. 1993, 47 (1), 558-561.

(37) Krukau, A. V.; Vydrov, O. A.; Izmaylov, A. F.; Scuseria, G. E. J. Chem. Phys. 2006, 125 (22), 224106.

(38) Tang, W.; Sanville, E.; Henkelman, G. J. Phys. Condens. Matter 2009, 21, 7.

(39) Henkelman, G.; Arnaldsson, A.; Jónsson, H. Comput. Mater. Sci. 2006, 36 (3), 354-360.

(40) Borlido, P.; Schmidt, J.; Huran, A. W.; Tran, F.; Marques, M. A. L.; Botti, S. npj Comput. Mater. 2020, 6, 96.

(41) Borlido, P.; Aull, T.; Huran, A. W.; Tran, F.; Marques, M. A. L.; Botti, S. J. Chem. Theory Comput. 2019, 15 (9), 5069-5079.

(42) Brazier, C. R.; Ellingboe, L. C.; Kinsey-Nielsen, S.; Bernath, P. F. J. Am. Chem. Soc. 1986, $108(9), 2126-2132$. 
(43) Yu, S.; Wang, J. G.; Sheridan, P. M.; Dick, M. J.; Bernath, P. F. J. Mol. Spectrosc. 2006, $240(1), 26-31$.

(44) Weinhold, F.; Carpenter, J. E. The Structure of Small Molecules and Ions; Naaman, R., Vager, Z., Eds.; Plenum, 1988.

(45) Rojas, H. N.; Godby, R. W.; Needs, R. J. Phys. Rev. Lett. 1995, 74 (10), 1827-1830.

(46) Grumet, M.; Liu, P.; Kaltak, M.; Klimeš, J.; Kresse, G. Phys. Rev. B 2018, 98 (15), 155143.

(47) Shishkin, M.; Kresse, G. Phys. Rev. B - Condens. Matter Mater. Phys. 2006, 74 (3), 035101.

(48) Notsu, H.; Yagi, I.; Tatsuma, T.; Tryk, D. A.; Fujishima, A. Electrochem. Solid-State Lett. 1999, 2 (10), 522-524.

(49) Kawarada, H. Surf. Sci. Rep. 1996, 26 (7), 205-259.

(50) Augenbraun, B. L.; Doyle, J. M.; Zelevinsky, T.; Kozyryev, I. Phys. Rev. X 2020, 10 (3), 31022.

(51) Fernando, W. T. M. L.; Ram, R. S.; O’Brien, L. C. O.; Bernath, P. F. J. Phys. Chem. 1991, 95 (9), 2665-2668.

(52) Bopegedera, A. M. R. P.; Brazier, C. R.; Bernath, P. F. J.Phys. Chem. 1987, 91 (11), 27792781.

(53) Aragonès, A. C.; Haworth, N. L.; Darwish, N.; Ciampi, S.; Bloomfield, N. J.; Wallace, G. G.; Diez-Perez, I.; Coote, M. L. Nature 2016, 531 (7592), 88-91. 
(54) Jaksch, D.; Cirac, J. I.; Zoller, P.; Rolston, S. L.; Côté, R.; Lukin, M. D. Phys. Rev. Lett. 2000, 85 (10), 2208-2211. 